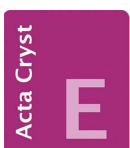

CRYSTALLOGRAPHIC COMMUNICATIONS

ISSN 2056-9890

Received 20 July 2016

Accepted 1 August 2016

Edited by T. J. Prior, University of Hull, England

Keywords: crystal structure; $\mathrm{CaZn}{ }_{2} \mathrm{Fe}\left(\mathrm{PO}_{4}\right)_{3}$; transition metal phosphate; solid-state reaction synthesis.

CCDC reference: 1497218

Supporting information: this article has supporting information at journals.iucr.org/e

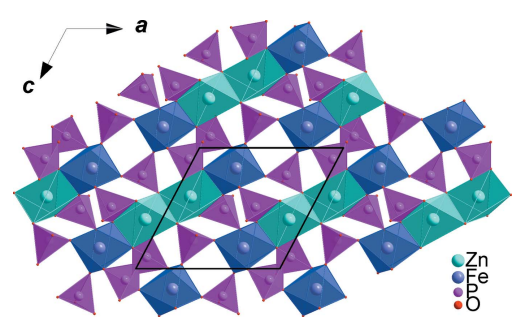

OPEN $\odot$ ACCESS

\section{Synthesis and crystal structure of calcium dizinc iron(III) tris(orthophosphate), $\mathrm{CaZn}{ }_{2} \mathrm{Fe}\left(\mathrm{PO}_{4}\right)_{3}$}

\author{
Jamal Khmiyas, ${ }^{*}$ Abderrazzak Assani, Mohamed Saadi and Lahcen El Ammari
}

Laboratoire de Chimie du Solide Appliquée, Faculty of Sciences, Mohammed V University in Rabat, Avenue Ibn Battouta, BP 1014, Rabat, Morocco. *Correspondence e-mail: j_khmiyas@yahoo.fr

Single crystals of the title compound, $\mathrm{CaZn} \mathrm{Fe}_{2}\left(\mathrm{PO}_{4}\right)_{3}$, were synthesized by conventional solid-state reaction. In the asymmetric unit, all atoms are located in fully occupied general positions of the $P 2_{1} / c$ space group. The zinc atoms are located on two crystallographically independent sites with tetrahedral and distorted triangular-based bipyramidal geometries. Two edge-sharing triangular bipyramidal $\mathrm{ZnO}_{5}$ units form a dimer, which is linked to slightly deformed $\mathrm{FeO}_{6}$ octahedra via a common edge. The resulting chains are interconnected through $\mathrm{PO}_{4}$ tetrahedra to form a layer perpendicular to the $b$ axis. Moreover, the remaining $\mathrm{PO}_{4}$ and $\mathrm{ZnO}_{4}$ tetrahedra are linked together through common vertices to form tapes parallel to the $c$ axis and surrounding a chain of $\mathrm{Ca}^{2+}$ cations to build a sheet, also perpendicular to the $b$ axis. The stacking of the two layers along the $b$ axis leads to the resulting three-dimensional framework, which defines channels in which the $\mathrm{Ca}^{2+}$ cations are located, each cation being coordinated by seven oxygen atoms.

\section{Chemical context}

Microporous compounds with an open anionic framework containing transition metals have been widely studied during recent years, especially iron phosphates, because of their potential applications in several fields such as gas sensing (Abdurahman et al., 2014), catalysis (Ai, 1999), as cathode materials for rechargeable lithium batteries (Masquelier et al., 1998), biocompatibility of glass fibres for tissue engineering (Ahmed et al., 2004), and immobilization of spent nuclear fuel (Mesko \& Day, 1999). Metal phosphates with an open framework can exhibit different architectures such as linearchain, layered and three-dimensional structures with channels or cavities where a variety of cations with different sizes, ratio and charges are accommodated. The occupancy of the allowed sites by cations can provide different properties such as remarkable flexibility, fast ionic conduction and low thermal expansion, mainly observed in the compounds belonging to the NASICON family with the general formula $M M^{\prime}{ }_{2} \mathrm{P}_{3} \mathrm{O}_{12}$ (where $M=$ alkali metal, alkaline-earth metal or a vacant site and $M^{\prime}=\mathrm{Zr}$, Ti, Hf, etc.; Senbhagaraman et al., 1993). In our previous hydrothermal investigations, a variety of compounds have been synthesized and characterized with different ratios of alkaline earth metal:P, viz. $\mathrm{Sr}_{2} \mathrm{Mn}_{3}\left(\mathrm{HPO}_{4}\right)_{2}\left(\mathrm{PO}_{4}\right)_{2}$ (Khmiyas et al., 2013), $\mathrm{BaMn}{ }_{2}^{\mathrm{II}} \mathrm{Mn}^{\mathrm{III}}\left(\mathrm{PO}_{4}\right)_{3}$ (Assani et al., 2013), $\mathrm{Mg}_{7}\left(\mathrm{PO}_{4}\right)_{2}\left(\mathrm{HPO}_{4}\right)_{4}$ (Assani et al., 2011). In this context, our interest is focused on the synthesis of new iron orthophosphates with an open-framework structure. Accordingly, we have succeeded in synthesizing and structurally characterizing a new calcium, zinc and iron-based open-framework phosphate, namely $\mathrm{CaZn}{ }_{2} \mathrm{Fe}\left(\mathrm{PO}_{4}\right)_{3}$. 


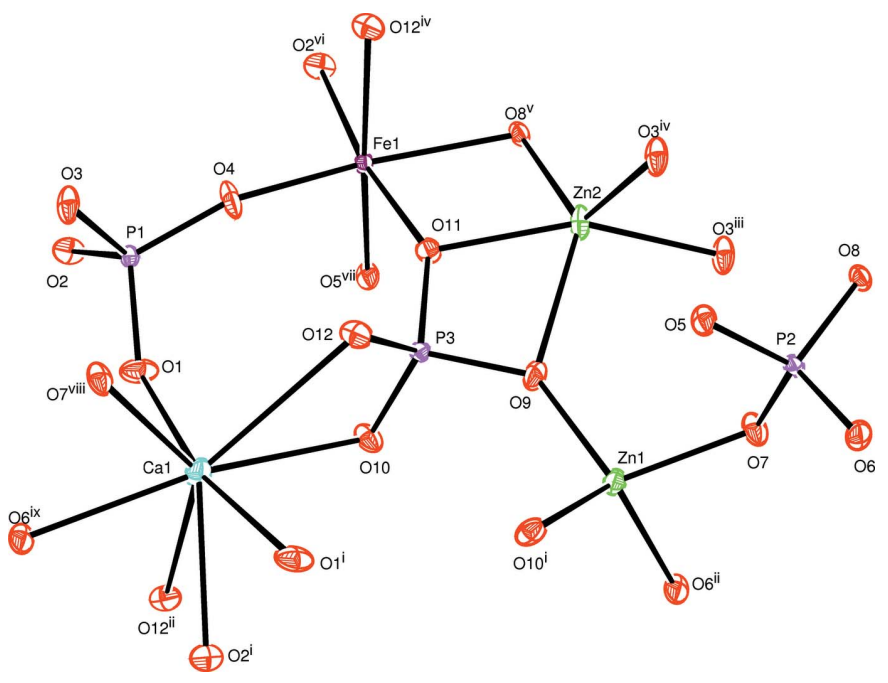

Figure 1

The principal building units in the structure of the title compound. Displacement ellipsoids are drawn at the $50 \%$ probability level. [Symmetry codes: (i) $x,-y+\frac{3}{2}, z-\frac{1}{2}$; (ii) $x,-y+\frac{3}{2}, z+\frac{1}{2}$; (iii) $x+1, y$, $z$; (iv) $-x+1,-y+1,-z+1$; (v) $-x+2,-y+1,-z+1$; (vi) $-x+1$, $-y+1,-z+2$; (vii) $x, y, z+1$; (viii) $x-1, y, z$; (ix) $x-1,-y+\frac{3}{2}, z+\frac{1}{2}$.]

\section{Structural commentary}

All atoms in asymmetric unit of the title compound occupy general positions of the $P 2_{1} / c$ space group. The refinement of this model was very easy and lead to an ordered structure in which the zinc cations occupy two sites with different environments. The coordination numbers of all cations were confirmed by bond-valence-sum calculations (Brown \& Altermatt, 1985). The obtained values for $\mathrm{Ca}^{\mathrm{II}+}, \mathrm{Zn}^{\mathrm{II}+}, \mathrm{Fe}^{\mathrm{III}+}$ and $\mathrm{P}^{\mathrm{V}+}$ are as expected, viz. Ca1 (1.93), $\mathrm{Zn} 1$ (2.00), $\mathrm{Zn} 2$ (1.91), Fe1 (3.04), P1 (5.11), P2 (4.97) and P3 (4.94). The crystal structure is build up from $\mathrm{PO}_{4}$ and ${\mathrm{Zn} 1 \mathrm{O}_{4}}_{4}$ tetrahedra, distorted triangular-based bipyramidal $\mathrm{Zn}_{2} \mathrm{O}_{5}$ and $\mathrm{FeO}_{6}$ octahedra, as shown in Fig. 1. The $\mathrm{FeO}_{6}$ octahedra are slightly deformed with $\mathrm{Fe}-\mathrm{O}$ distances varying from 1.8908 (8) to 2.1318 (8) $\AA$ and share a common edge with the highly distorted $\left[(\mathrm{Zn} 2)_{2} \mathrm{O}_{8}\right]$ dimer resulting from the edge-sharing of

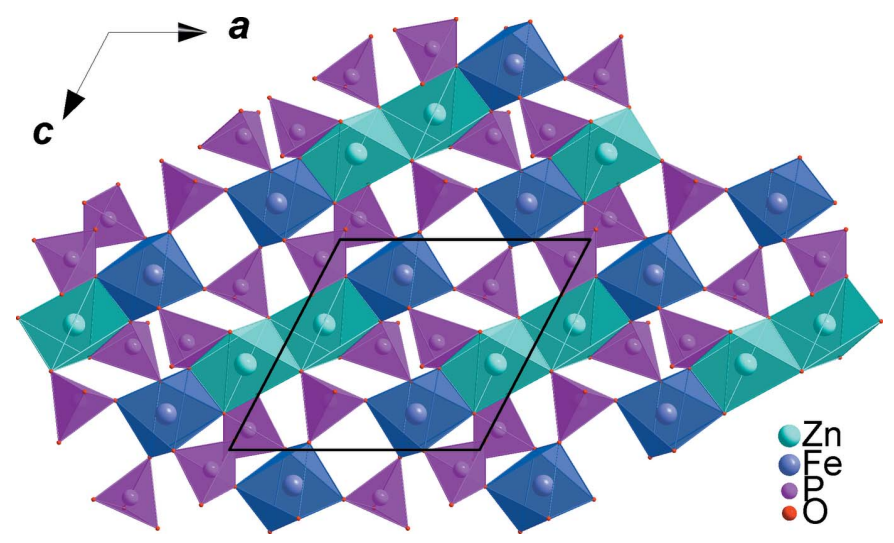

Figure 2

Edge-sharing triangular bipyramidal $\mathrm{ZnO}_{5}$ units linked to $\mathrm{FeO}_{6}$ octahedra and to $\mathrm{PO}_{4}$ tetrahedra, forming a layer perpendicular to the $b$ axis.

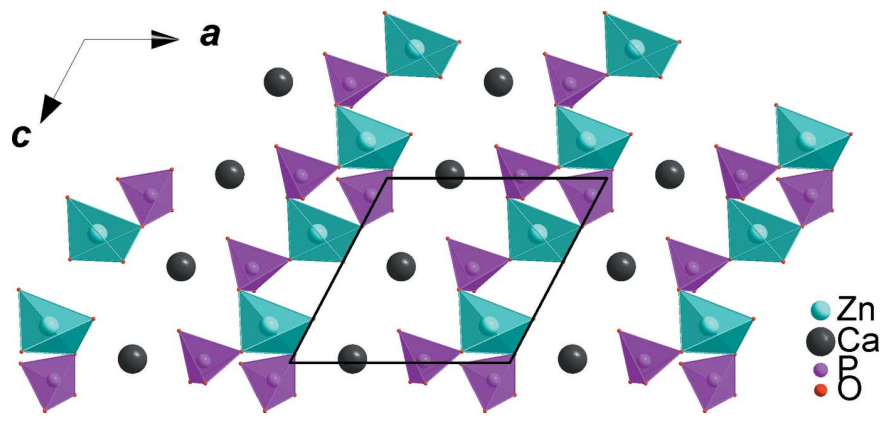

Figure 3

A layer perpendicular to the $b$ axis, resulting from the chains connected via vertices of the $\mathrm{ZnO}_{4}$ and $\mathrm{PO}_{4}$ tetrahedra.

two triangular-based bipyramidal ${\mathrm{Zn} 2 \mathrm{O}_{5}}_{5}$ units. Sequences of these polyhedra build chains interconnected by $\mathrm{PO}_{4}$ tetrahedra, forming a layer perpendicular to the $b$ axis, as shown in Fig. 2. The remaining $\mathrm{Zn} 1 \mathrm{O}_{4}$ tetrahedra are linked to irregular $\mathrm{PO}_{4}$ groups via common corners, forming tapes parallel to the $c$ axis, which are linked together by $\mathrm{Ca}^{2+}$ cations in sheets perpendicular to the $b$ axis (see Fig. 3). The obtained threedimensional framework shows one type of channel running along the [001] direction in which the $\mathrm{Ca}^{2+}$ cations are located, each being coordinated by seven oxygen atoms (Fig. 4).

\section{Database Survey}

The formula of the title compound, $\mathrm{CaZn} \mathrm{n}_{2} \mathrm{Fe}\left(\mathrm{PO}_{4}\right)_{3}$, is similar to some compounds with alluaudite structures, space group $C 2 / c$ or the $\alpha-\mathrm{CrPO}_{4}$ structure, space group Imma. However, its structure is different and to our knowledge there is no known isotypic structure. Crystals of $\mathrm{Ca} M_{2} \mathrm{Fe}\left(\mathrm{PO}_{4}\right)_{3}(M=\mathrm{Mg}$,

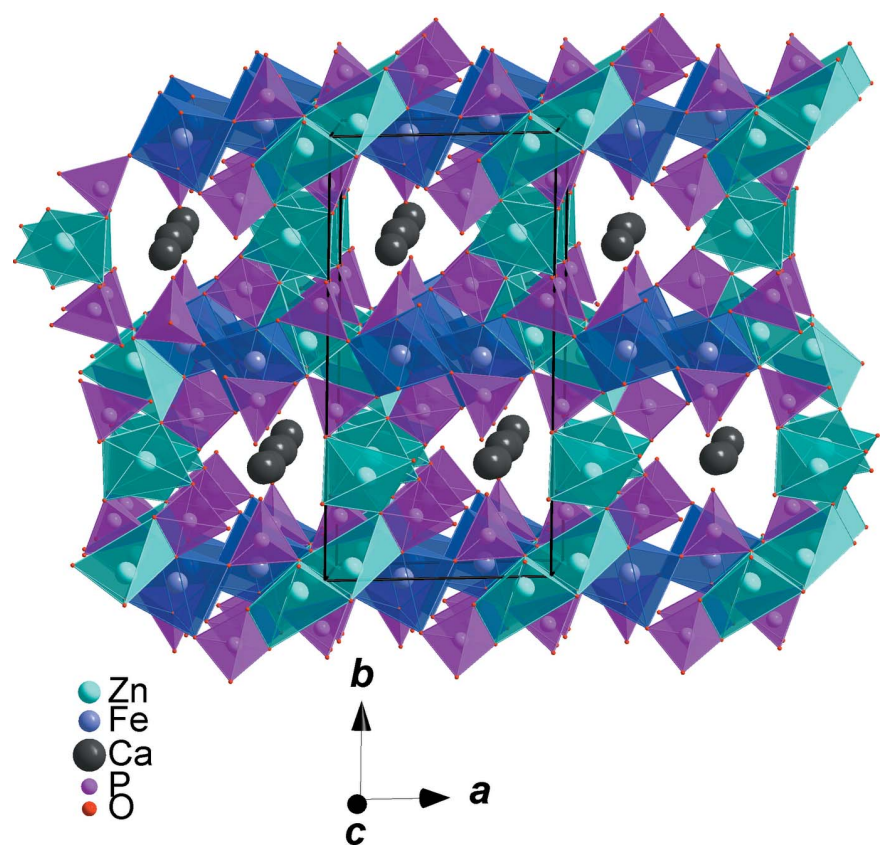

Figure 4

Polyhedral representation of $\mathrm{CaZn}{ }_{2} \mathrm{Fe}\left(\mathrm{PO}_{4}\right)_{3}$, showing the channels running along the [001] direction. 
Table 1

Experimental details.

\begin{tabular}{|c|c|}
\hline \multicolumn{2}{|l|}{ Crystal data } \\
\hline Chemical formula & $\mathrm{CaZn}{ }_{2} \mathrm{Fe}\left(\mathrm{PO}_{4}\right)_{3}$ \\
\hline$M_{\mathrm{r}}$ & 511.58 \\
\hline Crystal system, space group & Monoclinic, $P 2_{1} / c$ \\
\hline Temperature $(\mathrm{K})$ & 296 \\
\hline$a, b, c(\AA)$ & $8.5619(3), 15.2699(5), 8.1190(3)$ \\
\hline$\beta\left(^{\circ}\right)$ & $117.788(2)$ \\
\hline$V\left(\AA^{6}\right)$ & $939.06(6)$ \\
\hline$Z$ & 4 \\
\hline Radiation type & Mo $K \alpha$ \\
\hline$\mu\left(\mathrm{mm}^{-1}\right)$ & 7.72 \\
\hline Crystal size $(\mathrm{mm})$ & $0.30 \times 0.26 \times 0.18$ \\
\hline \multicolumn{2}{|l|}{ Data collection } \\
\hline Diffractometer & Bruker X8 APEX \\
\hline Absorption correction & $\begin{array}{l}\text { Multi-scan (SADABS; Krause et } \\
\text { al., 2015) }\end{array}$ \\
\hline$T_{\min }, T_{\max }$ & $0.600,0.747$ \\
\hline $\begin{array}{l}\text { No. of measured, independent and } \\
\text { observed }[I>2 \sigma(I)] \text { reflections }\end{array}$ & $54053,4985,4493$ \\
\hline$R_{\text {int }}$ & 0.033 \\
\hline$(\sin \theta / \lambda)_{\max }\left(\AA^{-1}\right)$ & 0.859 \\
\hline \multicolumn{2}{|l|}{ Refinement } \\
\hline$R\left[F^{2}>2 \sigma\left(F^{2}\right)\right], w R\left(F^{2}\right), S$ & $0.017,0.041,1.04$ \\
\hline No. of reflections & 4985 \\
\hline No. of parameters & 172 \\
\hline$\Delta \rho_{\max }, \Delta \rho_{\min }\left(\mathrm{e} \AA^{-3}\right)$ & $1.07,-0.78$ \\
\hline
\end{tabular}

Computer programs: APEX2 and SAINT (Bruker, 2009), SHELXT (Sheldrick, 2015a), SHELXL2014 (Sheldrick, 2015b), ORTEP-3 for Windows (Farrugia, 2012), DIAMOND (Brandenburg, 2006) and publCIF (Westrip, 2010).

$\mathrm{Co}, \mathrm{Ni}, \mathrm{Cu}$ ) compounds, which are predicted to have the same structures or isotypes are in preparation, while the structures of $\mathrm{Sr} M_{2} \mathrm{Fe}\left(\mathrm{PO}_{4}\right)_{3}(M=\mathrm{Co}, \mathrm{Ni})$ compounds are isotypic with $\alpha-\mathrm{CrPO}_{4}$ (Bouraima et al., 2016; Ouaatta et al., 2015). Mention may also be made of other similar compounds, for example the phosphates $\quad \mathrm{Na}_{2} \mathrm{Co}_{2} \mathrm{Fe}\left(\mathrm{PO}_{4}\right)_{3}, \quad \mathrm{NaCr}_{2} \mathrm{Zn}\left(\mathrm{PO}_{4}\right)_{3} \quad$ and $\mathrm{Na}_{1.66} \mathrm{Zn}_{1.66} \mathrm{Fe}_{1.34}\left(\mathrm{PO}_{4}\right)_{3}$ (Bouraima et al., 2015; Souiwa et al., 2015; Khmiyas et al., 2015) adopting the alluaudite structure type. In conclusion, we can say that the structure of this phosphate is similar to the alluaudite structure but with lower symmetry.

\section{Synthesis and crystallization}

Single crystals of $\mathrm{CaZn}{ }_{2} \mathrm{Fe}\left(\mathrm{PO}_{4}\right)_{3}$ were synthesized by a conventional solid-state method. Appropriate amounts of metal nitrate reagents, in the presence of $\mathrm{H}_{3} \mathrm{PO}_{4} 85 \mathrm{wt} \%$, were first dissolved in deionized water in the molar ratio Ca:Zn:Fe:P $=2: 2: 1: 3$ for $24 \mathrm{~h}$. Then, the resulting solution was evaporated to dryness. The powder residue was ground in an agate mortar and progressively heated in a platinum crucible at a heating rate of $141 \mathrm{~K} \mathrm{~h}^{-1}$ until melting occurred at $1283 \mathrm{~K}$. The melted product was cooled down at a rate of $5 \mathrm{~K} \mathrm{~h}^{-1}$. As result of the reaction, we obtained transparent crystals corresponding to the title compound $\mathrm{CaZn}{ }_{2} \mathrm{Fe}\left(\mathrm{PO}_{4}\right)_{3}$.

\section{Refinement}

Crystal data, data collection and structure refinement details are summarized in Table 1. The reflections (202) and (330) probably affected by the beam-stop were omitted from the refinement. The maximum and minimum electron densities in the final Fourier map are at 0.56 and $0.44 \AA$ from $\mathrm{Ca} 1$ and $\mathrm{Zn} 2$, respectively.

\section{Acknowledgements}

The authors thank the Unit of Support for Technical and Scientific Research (UATRS, CNRST) for the X-ray measurements and Mohammed V University in Rabat, Morocco, for financial support.

\section{References}

Abdurahman, A., Nizamidin, P. \& Yimit, A. (2014). Mater. Sci. Semicond. Process. 22, 21-27.

Ahmed, I., Collins, C. A., Lewis, M. P., Olsen, I. \& Knowles, J. C. (2004). Biomaterials, 25, 3223-3232.

Ai, M. (1999). Catal. Today, 52, 65-69.

Assani, A., Saadi, M., Alhakmi, G., Houmadi, E. \& El Ammari, L. (2013). Acta Cryst. E69, i60.

Assani, A., Saadi, M., Zriouil, M. \& El Ammari, L. (2011). Acta Cryst. E67, i52.

Bouraima, A., Assani, A., Saadi, M., Makani, T. \& El Ammari, L. (2015). Acta Cryst. E71, 558-560.

Bouraima, A., Makani, T., Assani, A., Saadi, M. \& El Ammari, L. (2016). Acta Cryst. E72, 1143-1146.

Brandenburg, K. (2006). DIAMOND. Crystal Impact GbR, Bonn, Germany.

Brown, I. D. \& Altermatt, D. (1985). Acta Cryst. B41, 244-247.

Bruker (2009). APEX2 and SAINT. Bruker AXS Inc., Madison, Wisconsin, USA.

Farrugia, L. J. (2012). J. Appl. Cryst. 45, 849-854.

Khmiyas, J., Assani, A., Saadi, M. \& El Ammari, L. (2013). Acta Cryst. E69, i50.

Khmiyas, J., Assani, A., Saadi, M. \& El Ammari, L. (2015). Acta Cryst. E71, 690-692.

Krause, L., Herbst-Irmer, R., Sheldrick, G. M. \& Stalke, D. (2015). J. Appl. Cryst. 48, 3-10.

Masquelier, C., Padhi, A. K., Nanjundaswamy, K. S. \& Goodenough, J. B. (1998). J. Solid State Chem. 135, 228-234.

Mesko, M. G. \& Day, D. E. (1999). J. Nucl. Mater. 273, 27-36.

Ouaatta, S., Assani, A., Saadi, M. \& El Ammari, L. (2015). Acta Cryst. E71, 1255-1258.

Senbhagaraman, S., Guru Rowb, T. N. \& Umarji, A. M. (1993). J. Mater. Chem. 3, 309-314.

Sheldrick, G. M. (2015a). Acta Cryst. A71, 3-8.

Sheldrick, G. M. (2015b). Acta Cryst. C71, 3-8.

Souiwa, K., Hidouri, M., Toulemonde, O., Duttine, M. \& Ben Amara, M. (2015). J. Alloys Compd, 627, 153-160.

Westrip, S. P. (2010). J. Appl. Cryst. 43, 920-925. 


\section{supporting information}

Acta Cryst. (2016). E72, 1260-1262 [https://doi.org/10.1107/S2056989016012421]

Synthesis and crystal structure of calcium dizinc iron(III) tris(orthophosphate), $\mathrm{CaZn}{ }_{2} \mathrm{Fe}\left(\mathrm{PO}_{4}\right)_{3}$

Jamal Khmiyas, Abderrazzak Assani, Mohamed Saadi and Lahcen El Ammari

Computing details

Data collection: APEX2 (Bruker, 2009); cell refinement: SAINT (Bruker, 2009); data reduction: SAINT (Bruker, 2009); program(s) used to solve structure: SHELXT (Sheldrick, 2015a); program(s) used to refine structure: SHELXL2014 (Sheldrick, 2015b); molecular graphics: ORTEP-3 for Windows (Farrugia, 2012) and DIAMOND (Brandenburg, 2006); software used to prepare material for publication: publCIF (Westrip, 2010).

Calcium dizinc iron(III) tris(orthophosphate)

Crystal data

$\mathrm{CaZn} 2 \mathrm{Fe}\left(\mathrm{PO}_{4}\right)_{3}$

$M_{r}=511.58$

Monoclinic, $P 2_{1} / c$

$a=8.5619(3) \AA$

$b=15.2699(5) \AA$

$c=8.1190(3) \AA$

$\beta=117.788(2)^{\circ}$

$V=939.06(6) \AA^{3}$

$Z=4$

\section{Data collection}

Bruker X8 APEX diffractometer

Radiation source: fine-focus sealed tube Graphite monochromator $\varphi$ and $\omega$ scans

Absorption correction: multi-scan

(SADABS; Krause et al., 2015)

$T_{\min }=0.600, T_{\max }=0.747$

Refinement

Refinement on $F^{2}$

Least-squares matrix: full

$R\left[F^{2}>2 \sigma\left(F^{2}\right)\right]=0.017$

$w R\left(F^{2}\right)=0.041$

$S=1.04$

4985 reflections

172 parameters
$F(000)=988$

$D_{\mathrm{x}}=3.618 \mathrm{Mg} \mathrm{m}^{-3}$

Mo $K \alpha$ radiation, $\lambda=0.71073 \AA$

Cell parameters from 4985 reflections

$\theta=2.7-37.6^{\circ}$

$\mu=7.72 \mathrm{~mm}^{-1}$

$T=296 \mathrm{~K}$

Block, black

$0.30 \times 0.26 \times 0.18 \mathrm{~mm}$

54053 measured reflections

4985 independent reflections

4493 reflections with $I>2 \sigma(I)$

$R_{\text {int }}=0.033$

$\theta_{\max }=37.6^{\circ}, \theta_{\min }=2.7^{\circ}$

$h=-14 \rightarrow 14$

$k=-26 \rightarrow 26$

$l=-10 \rightarrow 13$

0 restraints

$w=1 /\left[\sigma^{2}\left(F_{\mathrm{o}}^{2}\right)+(0.0174 P)^{2}+0.7655 P\right]$

where $P=\left(F_{\mathrm{o}}{ }^{2}+2 F_{\mathrm{c}}{ }^{2}\right) / 3$

$(\Delta / \sigma)_{\max }=0.003$

$\Delta \rho_{\max }=1.07 \mathrm{e} \AA^{-3}$

$\Delta \rho_{\min }=-0.78$ e $\AA^{-3}$ 


\section{Special details}

Geometry. All esds (except the esd in the dihedral angle between two 1.s. planes) are estimated using the full covariance matrix. The cell esds are taken into account individually in the estimation of esds in distances, angles and torsion angles; correlations between esds in cell parameters are only used when they are defined by crystal symmetry. An approximate (isotropic) treatment of cell esds is used for estimating esds involving l.s. planes.

Fractional atomic coordinates and isotropic or equivalent isotropic displacement parameters $\left(\AA^{2}\right)$

\begin{tabular}{lllll}
\hline & $x$ & $y$ & $z$ & $U_{\text {iso }} * / U_{\text {eq }}$ \\
\hline Zn1 & $0.81685(2)$ & $0.73588(2)$ & $0.30142(2)$ & $0.00740(3)$ \\
Zn2 & $0.88412(2)$ & $0.52265(2)$ & $0.59417(2)$ & $0.01026(3)$ \\
Fe1 & $0.67075(2)$ & $0.49009(2)$ & $0.83003(2)$ & $0.00500(3)$ \\
Ca1 & $0.27619(3)$ & $0.75762(2)$ & $0.47919(3)$ & $0.01070(4)$ \\
P1 & $0.29665(3)$ & $0.58370(2)$ & $0.77261(4)$ & $0.00503(4)$ \\
P2 & $0.96807(3)$ & $0.62244(2)$ & $0.09438(4)$ & $0.00499(4)$ \\
P3 & $0.60325(3)$ & $0.64096(2)$ & $0.49014(4)$ & $0.00491(4)$ \\
O1 & $0.29942(13)$ & $0.67932(6)$ & $0.72349(13)$ & $0.01271(15)$ \\
O2 & $0.30273(12)$ & $0.58596(6)$ & $0.96463(12)$ & $0.01006(14)$ \\
O3 & $0.12207(10)$ & $0.54161(6)$ & $0.62684(12)$ & $0.01114(15)$ \\
O4 & $0.43948(11)$ & $0.52893(6)$ & $0.76475(14)$ & $0.01312(16)$ \\
O5 & $0.77926(10)$ & $0.58832(5)$ & $0.00379(12)$ & $0.00895(13)$ \\
O6 & $1.00136(11)$ & $0.67493(6)$ & $-0.04745(13)$ & $0.01045(14)$ \\
O7 & $1.00262(11)$ & $0.68143(6)$ & $0.26134(13)$ & $0.01055(14)$ \\
O8 & $1.09932(10)$ & $0.54481(5)$ & $0.17408(12)$ & $0.00724(13)$ \\
O9 & $0.75426(11)$ & $0.65280(6)$ & $0.43941(13)$ & $0.01160(15)$ \\
O10 & $0.59377(10)$ & $0.71947(6)$ & $0.60355(12)$ & $0.00974(14)$ \\
O11 & $0.66602(11)$ & $0.55677(5)$ & $0.61102(12)$ & $0.00822(13)$ \\
O12 & $0.41993(10)$ & $0.62799(6)$ & $0.32620(12)$ & $0.01012(14)$ \\
& & & &
\end{tabular}

Atomic displacement parameters $\left(\AA^{2}\right)$

\begin{tabular}{lllllll}
\hline & $U^{11}$ & $U^{22}$ & $U^{33}$ & $U^{12}$ & $U^{13}$ & $U^{23}$ \\
\hline Zn1 & $0.00645(5)$ & $0.00817(5)$ & $0.00801(6)$ & $0.00013(4)$ & $0.00374(4)$ & $0.00104(4)$ \\
Zn2 & $0.00760(5)$ & $0.01471(6)$ & $0.01073(6)$ & $0.00042(4)$ & $0.00616(4)$ & $-0.00080(5)$ \\
Fe1 & $0.00454(5)$ & $0.00602(6)$ & $0.00488(6)$ & $0.00044(4)$ & $0.00256(4)$ & $0.00061(4)$ \\
Ca1 & $0.01029(8)$ & $0.01095(9)$ & $0.01178(10)$ & $0.00175(6)$ & $0.00591(7)$ & $0.00601(7)$ \\
P1 & $0.00467(9)$ & $0.00607(10)$ & $0.00452(10)$ & $0.00011(7)$ & $0.00229(8)$ & $-0.00022(8)$ \\
P2 & $0.00441(9)$ & $0.00482(10)$ & $0.00581(11)$ & $0.00038(7)$ & $0.00243(8)$ & $-0.00009(8)$ \\
P3 & $0.00448(9)$ & $0.00500(10)$ & $0.00483(10)$ & $0.00004(7)$ & $0.00182(8)$ & $0.00031(8)$ \\
O1 & $0.0222(4)$ & $0.0076(3)$ & $0.0109(4)$ & $-0.0001(3)$ & $0.0098(3)$ & $0.0019(3)$ \\
O2 & $0.0169(3)$ & $0.0093(3)$ & $0.0063(3)$ & $0.0009(3)$ & $0.0073(3)$ & $0.0011(3)$ \\
O3 & $0.0051(3)$ & $0.0176(4)$ & $0.0102(4)$ & $-0.0030(3)$ & $0.0030(3)$ & $-0.0063(3)$ \\
O4 & $0.0065(3)$ & $0.0172(4)$ & $0.0157(4)$ & $0.0026(3)$ & $0.0052(3)$ & $-0.0045(3)$ \\
O5 & $0.0056(3)$ & $0.0100(3)$ & $0.0101(3)$ & $-0.0016(2)$ & $0.0028(2)$ & $-0.0034(3)$ \\
O6 & $0.0097(3)$ & $0.0107(3)$ & $0.0132(4)$ & $0.0029(3)$ & $0.0072(3)$ & $0.0061(3)$ \\
O7 & $0.0079(3)$ & $0.0122(3)$ & $0.0115(4)$ & $-0.0005(2)$ & $0.0045(3)$ & $-0.0062(3)$ \\
O8 & $0.0065(3)$ & $0.0073(3)$ & $0.0088(3)$ & $0.0026(2)$ & $0.0043(2)$ & $0.0020(2)$ \\
O9 & $0.0111(3)$ & $0.0118(3)$ & $0.0161(4)$ & $0.0008(3)$ & $0.0098(3)$ & $0.0042(3)$
\end{tabular}




\begin{tabular}{lllllll}
$\mathrm{O} 10$ & $0.0076(3)$ & $0.0091(3)$ & $0.0103(3)$ & $0.0006(2)$ & $0.0024(3)$ & $-0.0040(3)$ \\
$\mathrm{O} 11$ & $0.0100(3)$ & $0.0080(3)$ & $0.0082(3)$ & $0.0028(2)$ & $0.0055(3)$ & $0.0039(3)$ \\
$\mathrm{O} 12$ & $0.0068(3)$ & $0.0086(3)$ & $0.0097(3)$ & $-0.0002(2)$ & $-0.0006(3)$ & $-0.0020(3)$ \\
\hline
\end{tabular}

Geometric parameters $\left(\AA,{ }^{\circ}\right)$

\begin{tabular}{|c|c|c|c|}
\hline $\mathrm{Zn} 1-\mathrm{O} 9$ & $1.9266(9)$ & $\mathrm{Ca} 1-\mathrm{O} 2^{\mathrm{i}}$ & $2.4075(9)$ \\
\hline $\mathrm{Zn} 1-\mathrm{O} 7$ & $1.9518(8)$ & $\mathrm{Ca} 1-\mathrm{O} 7^{\mathrm{viii}}$ & $2.4709(9)$ \\
\hline $\mathrm{Zn} 1-\mathrm{O} 10^{\mathrm{i}}$ & $1.9578(8)$ & $\mathrm{Ca} 1-\mathrm{O} 6^{\mathrm{ix}}$ & $2.4840(9)$ \\
\hline $\mathrm{Zn} 1-\mathrm{O}^{\mathrm{ii}}$ & $2.0120(8)$ & $\mathrm{Ca} 1-\mathrm{O} 10$ & $2.4885(8)$ \\
\hline $\mathrm{Zn} 2-\mathrm{O} 3^{\mathrm{iii}}$ & $1.9496(8)$ & $\mathrm{Ca} 1-\mathrm{O} 12$ & $2.8984(9)$ \\
\hline $\mathrm{Zn} 2-\mathrm{O} 11$ & $2.0038(8)$ & $\mathrm{P} 1-\mathrm{O} 4$ & $1.5073(9)$ \\
\hline $\mathrm{Zn} 2-\mathrm{O}^{\mathrm{iv}}$ & $2.0241(9)$ & $\mathrm{P} 1-\mathrm{O} 1$ & $1.5166(9)$ \\
\hline $\mathrm{Zn} 2-\mathrm{O}^{\mathrm{v}}$ & $2.0911(8)$ & $\mathrm{P} 1-\mathrm{O} 2$ & $1.5358(9)$ \\
\hline $\mathrm{Zn} 2-\mathrm{O} 9$ & $2.3371(9)$ & $\mathrm{P} 1-\mathrm{O} 3$ & $1.5487(8)$ \\
\hline $\mathrm{Fe} 1-\mathrm{O} 4$ & $1.8908(8)$ & $\mathrm{P} 2-\mathrm{O} 5$ & $1.5222(8)$ \\
\hline $\mathrm{Fe} 1-\mathrm{O} 2^{\mathrm{vi}}$ & $1.9561(9)$ & $\mathrm{P} 2-\mathrm{O} 6$ & $1.5348(9)$ \\
\hline $\mathrm{Fe} 1-\mathrm{O} 5^{\mathrm{vii}}$ & $1.9700(8)$ & $\mathrm{P} 2-\mathrm{O} 7$ & $1.5371(9)$ \\
\hline $\mathrm{Fe} 1-\mathrm{O} 11$ & $2.0330(8)$ & $\mathrm{P} 2-\mathrm{O} 8$ & $1.5519(8)$ \\
\hline $\mathrm{Fe} 1-\mathrm{O}^{\mathrm{v}}$ & $2.0547(8)$ & $\mathrm{P} 3-\mathrm{O} 12$ & $1.5253(8)$ \\
\hline $\mathrm{Fe} 1-\mathrm{O} 12^{\mathrm{iv}}$ & $2.1318(8)$ & $\mathrm{P} 3-\mathrm{O} 10$ & $1.5365(9)$ \\
\hline $\mathrm{Ca} 1-\mathrm{O} 1$ & $2.2439(9)$ & $\mathrm{P} 3-\mathrm{O} 9$ & $1.5396(9)$ \\
\hline $\mathrm{Ca} 1-\mathrm{O}^{\mathrm{i}}$ & $2.3795(10)$ & $\mathrm{P} 3-\mathrm{O} 11$ & $1.5534(8)$ \\
\hline $\mathrm{O} 9-\mathrm{Zn} 1-\mathrm{O} 7$ & $106.60(4)$ & $\mathrm{O} 2^{\mathrm{i}}-\mathrm{Ca} 1-\mathrm{O}^{\mathrm{ix}}$ & $72.04(3)$ \\
\hline $\mathrm{O} 9-\mathrm{Zn} 1-\mathrm{O} 10^{\mathrm{i}}$ & $106.09(4)$ & $\mathrm{O} 7^{\text {viii }-\mathrm{Ca} 1-\mathrm{O}^{\mathrm{ix}}}$ & $65.76(3)$ \\
\hline $\mathrm{O} 7-\mathrm{Zn} 1-\mathrm{O} 10^{\mathrm{i}}$ & $124.80(4)$ & $\mathrm{O} 1-\mathrm{Ca} 1-\mathrm{O} 10$ & $83.50(3)$ \\
\hline $\mathrm{O} 9-\mathrm{Zn} 1-\mathrm{O}^{\mathrm{ii}}$ & $116.31(4)$ & $\mathrm{O} 11^{\mathrm{i}}-\mathrm{Ca} 1-\mathrm{O} 10$ & $85.92(3)$ \\
\hline $\mathrm{O} 7-\mathrm{Zn} 1-\mathrm{O}^{\mathrm{ii}}$ & $85.46(3)$ & $\mathrm{O} 2 \mathrm{i}-\mathrm{Ca} 1-\mathrm{O} 10$ & $98.16(3)$ \\
\hline 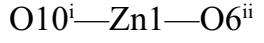 & $116.93(4)$ & $\mathrm{O} 7^{\text {viii-Ca1-O10 }}$ & $132.22(3)$ \\
\hline 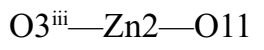 & $154.16(4)$ & $\mathrm{O} 6^{\mathrm{ix}}-\mathrm{Ca} 1-\mathrm{O} 10$ & $160.73(3)$ \\
\hline 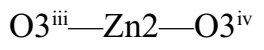 & $77.67(4)$ & $\mathrm{O} 1-\mathrm{Ca} 1-\mathrm{O} 12$ & $97.73(3)$ \\
\hline $\mathrm{O} 11-\mathrm{Zn} 2-\mathrm{O}^{\mathrm{iv}}$ & $123.13(3)$ & $\mathrm{O} 1-\mathrm{Ca} 1-\mathrm{O} 12$ & $71.19(3)$ \\
\hline 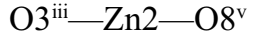 & $108.82(4)$ & $\mathrm{O} 22^{\mathrm{i}}-\mathrm{Ca} 1-\mathrm{O} 12$ & $126.00(3)$ \\
\hline $\mathrm{O} 11-\mathrm{Zn} 2-\mathrm{O}^{\mathrm{v}}$ & $75.00(3)$ & O7 $7^{\text {viii_-Ca1-O12 }}$ & $79.74(3)$ \\
\hline $\mathrm{O}^{\mathrm{iv}}-\mathrm{Zn} 2-\mathrm{O}^{\mathrm{v}}$ & $121.44(4)$ & $\mathrm{O} 6^{\mathrm{ix}}-\mathrm{Ca} 1-\mathrm{O} 12$ & $145.10(3)$ \\
\hline $\mathrm{O} 3{ }^{\mathrm{iii}}-\mathrm{Zn} 2-\mathrm{O} 9$ & $98.73(4)$ & $\mathrm{O} 10-\mathrm{Ca} 1-\mathrm{O} 12$ & $53.99(3)$ \\
\hline $\mathrm{O} 11-\mathrm{Zn} 2-\mathrm{O} 9$ & $65.84(3)$ & $\mathrm{O} 1-\mathrm{Ca} 1-\mathrm{O} 12^{\mathrm{ii}}$ & $69.93(3)$ \\
\hline $\mathrm{O}^{\text {iv }}-\mathrm{Zn} 2-\mathrm{O} 9$ & $97.26(4)$ & $\mathrm{O} 1^{\mathrm{i}}-\mathrm{Ca} 1-\mathrm{O} 12^{\mathrm{ii}}$ & $114.39(3)$ \\
\hline $\mathrm{O} 8^{\mathrm{v}}-\mathrm{Zn} 2-\mathrm{O} 9$ & $135.92(3)$ & $\mathrm{O} 2^{\mathrm{i}}-\mathrm{Ca} 1-\mathrm{O} 12^{\mathrm{ii}}$ & $57.96(3)$ \\
\hline $\mathrm{O} 4-\mathrm{Fe} 1-\mathrm{O} 2^{\mathrm{vi}}$ & $96.63(4)$ & $\mathrm{O} 7^{\mathrm{viii}}-\mathrm{Ca} 1-\mathrm{O} 12^{\mathrm{ii}}$ & $140.52(3)$ \\
\hline $\mathrm{O} 4-\mathrm{Fe} 1-\mathrm{O} 5^{\text {vii }}$ & $92.55(4)$ & $\mathrm{O}^{\mathrm{ix}}-\mathrm{Ca} 1-\mathrm{O} 12^{\mathrm{ii}}$ & $78.49(3)$ \\
\hline $\mathrm{O} 2^{\mathrm{vi}}-\mathrm{Fe} 1-\mathrm{O}^{\mathrm{vii}}$ & $90.75(4)$ & $\mathrm{O} 10-\mathrm{Ca} 1-\mathrm{O} 12^{\mathrm{ii}}$ & $82.24(3)$ \\
\hline $\mathrm{O} 4-\mathrm{Fe} 1-\mathrm{O} 11$ & $90.37(4)$ & $\mathrm{O} 12-\mathrm{Ca} 1-\mathrm{O} 12^{\mathrm{ii}}$ & $135.98(3)$ \\
\hline $\mathrm{O} 2^{\mathrm{vi}}-\mathrm{Fe} 1-\mathrm{O} 11$ & $171.82(3)$ & $\mathrm{O} 4-\mathrm{P} 1-\mathrm{O} 1$ & $114.24(6)$ \\
\hline $\mathrm{O}^{\mathrm{vii}}-\mathrm{Fe} 1-\mathrm{O} 11$ & $93.18(4)$ & $\mathrm{O} 4-\mathrm{P} 1-\mathrm{O} 2$ & $114.28(5)$ \\
\hline $\mathrm{O} 4-\mathrm{Fe} 1-\mathrm{O}^{\mathrm{v}}$ & $164.34(4)$ & $\mathrm{O} 1-\mathrm{P} 1-\mathrm{O} 2$ & $104.36(5)$ \\
\hline $\mathrm{O} 2^{\mathrm{vi}}-\mathrm{Fe} 1-\mathrm{O} 8^{\mathrm{v}}$ & $97.38(3)$ & $\mathrm{O} 4-\mathrm{P} 1-\mathrm{O} 3$ & $104.50(5)$ \\
\hline
\end{tabular}




\begin{tabular}{|c|c|c|c|}
\hline $\mathrm{O} 5^{\mathrm{vii}-\mathrm{Fe} 1-\mathrm{O}^{\mathrm{v}}}$ & $94.22(3)$ & $\mathrm{O} 1-\mathrm{P} 1-\mathrm{O} 3$ & $109.05(5)$ \\
\hline $\mathrm{O} 11-\mathrm{Fe} 1-\mathrm{O}^{\mathrm{v}}$ & $75.18(3)$ & $\mathrm{O} 2-\mathrm{P} 1-\mathrm{O} 3$ & $110.42(5)$ \\
\hline $\mathrm{O} 4-\mathrm{Fe} 1-\mathrm{O} 12^{\mathrm{iv}}$ & $93.16(4)$ & $\mathrm{O} 5-\mathrm{P} 2-\mathrm{O} 6$ & $110.07(5)$ \\
\hline $\mathrm{O} 2^{\mathrm{vi}}-\mathrm{Fe} 1-\mathrm{O} 12^{\mathrm{iv}}$ & $82.55(4)$ & $\mathrm{O} 5-\mathrm{P} 2-\mathrm{O} 7$ & $110.55(5)$ \\
\hline $\mathrm{O} 5^{\mathrm{vii}}-\mathrm{Fe} 1-\mathrm{O} 12^{\mathrm{iv}}$ & $171.65(3)$ & $\mathrm{O} 6-\mathrm{P} 2-\mathrm{O} 7$ & $109.20(5)$ \\
\hline $\mathrm{O} 11-\mathrm{Fe} 1-\mathrm{O} 12^{\mathrm{iv}}$ & $92.86(4)$ & $\mathrm{O} 5-\mathrm{P} 2-\mathrm{O} 8$ & $109.84(5)$ \\
\hline $\mathrm{O} 8^{\mathrm{v}}-\mathrm{Fe} 1-\mathrm{O} 12^{\mathrm{iv}}$ & $81.77(3)$ & $\mathrm{O} 6-\mathrm{P} 2-\mathrm{O} 8$ & $111.11(5)$ \\
\hline $\mathrm{O} 1-\mathrm{Ca} 1-\mathrm{O} 1^{\mathrm{i}}$ & $167.91(4)$ & $\mathrm{O} 7-\mathrm{P} 2-\mathrm{O} 8$ & $106.00(5)$ \\
\hline $\mathrm{O} 1-\mathrm{Ca} 1-\mathrm{O} 2^{\mathrm{i}}$ & $126.91(3)$ & $\mathrm{O} 12-\mathrm{P} 3-\mathrm{O} 10$ & $107.69(5)$ \\
\hline $\mathrm{O} 1^{\mathrm{i}}-\mathrm{Ca} 1-\mathrm{O} 2^{\mathrm{i}}$ & $60.49(3)$ & $\mathrm{O} 12-\mathrm{P} 3-\mathrm{O} 9$ & $115.63(5)$ \\
\hline $\mathrm{O} 1-\mathrm{Ca} 1-\mathrm{O}^{\text {viii }}$ & $92.62(3)$ & $\mathrm{O} 10-\mathrm{P} 3-\mathrm{O} 9$ & $110.75(5)$ \\
\hline $\mathrm{O} 1^{\mathrm{i}}-\mathrm{Ca} 1-\mathrm{O} 7^{\text {viii }}$ & $90.17(3)$ & $\mathrm{O} 12-\mathrm{P} 3-\mathrm{O} 11$ & $110.86(5)$ \\
\hline $\mathrm{O} 2^{\mathrm{i}}-\mathrm{Ca} 1-\mathrm{O} 7^{\mathrm{viii}}$ & $120.77(3)$ & $\mathrm{O} 10-\mathrm{P} 3-\mathrm{O} 11$ & $111.50(5)$ \\
\hline $\mathrm{O} 1-\mathrm{Ca} 1-\mathrm{O}^{\mathrm{ix}}$ & $89.31(3)$ & $\mathrm{O} 9-\mathrm{P} 3-\mathrm{O} 11$ & $100.36(5)$ \\
\hline $\mathrm{O} 1^{\mathrm{i}}-\mathrm{Ca} 1-\mathrm{O}^{\mathrm{ix}}$ & 102.55 & & \\
\hline
\end{tabular}

Symmetry codes: (i) $x,-y+3 / 2, z-1 / 2$; (ii) $x,-y+3 / 2, z+1 / 2$; (iii) $x+1, y, z$; (iv) $-x+1,-y+1,-z+1$; (v) $-x+2,-y+1,-z+1$; (vi) $-x+1,-y+1,-z+2$; (vii) $x, y$, $z+1$; (viii) $x-1, y, z$; (ix) $x-1,-y+3 / 2, z+1 / 2$. 